\title{
Ineffective Cricoid Pressure: The Critical Role of Formalised Training
}

\author{
Daniel D. Moos* \\ Staff Anesthetist, Kearney Anesthesia Associates, P.C., Kearney, Nebraska, USA; Adjunct Faculty, \\ Bryan/LGH College of Health Sciences, School of Nurse Anesthesia, Lincoln, Nebraska, USA
}

\begin{abstract}
The application of cricoid pressure may help reduce the incidence of pulmonary aspiration in at-risk patients. Research has shown that most clinicians have a cognitive deficit concerning the correct amount of pressure to apply, anatomical landmarks and apply the incorrect amount of pressure. Too much pressure may lead to an airway obstruction or distorted view for laryngoscopy. Too little pressure may lead to pulmonary aspiration. It is imperative that clinicians who apply cricoid pressure have a thorough knowledge of basic anatomy, have a basic understanding of regurgitation/aspiration, indications, complications, contraindications and common problems/mistakes when applying cricoid pressure. Various teaching strategies to help the clinician apply correct and effective cricoid pressure have been described in the literature. All clinicians should be formally trained in the proper technique for the application of cricoid pressure.
\end{abstract}

Key words: cricoid pressure; Sellick's manoeuvre; teaching techniques

\section{INTRODUCTION}

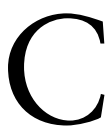
ricoid pressure is a thoroughly English technique. It was first described in 1774 . Dr. Munro, an English physician, described the use of cricoid pressure to prevent gastric distention during the

"The author is in private practice with Kearney Anesthesia Associates, P.C., in Kearney, Nebraska. In addition he is adjunct faculty for Bryan/LGH College of Health Sciences, School of Nurse Anesthesia in Lincoln, Nebraska. He is involved with the clinical and didactic instruction of nurse anaesthesia students. resuscitation of drowning victims. It was not until 1961 that cricoid pressure would be applied to dominion of anaesthesia by B.A. Sellick, an English anaesthesiologist. Dr. Sellick suggested that cricoid pressure should be used to prevent gastric insufflation of air during positive pressure ventilation and to prevent passive regurgitation/aspiration of gastric contents [Salem et al, 1974]. The term 'Sellick's manoeuvre' is synonymous with cricoid pressure. In addition R.G. Vanner, an English anaesthesiologist, has researched and published extensively on cricoid pressure.

The application of cricoid pressure to reduce the incidence of pulmonary aspiration, in at-risk patients, has become a standard technique for anaesthesia providers throughout the world. Improper application of cricoid pressure may lead to increased morbidity and mortality. If the amount of cricoid pressure is insufficient, the patient may be at risk for aspiration. If the amount of cricoid pressure is excessive, the patient's airway may be distorted leading to a challenging intubation or obstructed airway. Failure to identify the correct anatomical landmarks may lead to ineffective application of cricoid pressure. Clinicians responsible for the application of cricoid pressure should have a thorough knowledge of basic anatomy, basic understanding of regurgitation/ aspiration, indications, complications, contraindications, common problems/mistakes and teaching strategies to learn how to apply correct and effective cricoid pressure.

\section{BASIC OESOPHAGEAL AND LARYNGOTRACHEAL ANATOMY AS IT RELATES TO CRICOID PRESSURE}

The oesophagus extends from the pharynx, at the level of the 6th cervical vertebrae, to the stomach. Anatomically it is divided into the upper oesophageal sphincter (UES), 
the body of the oesophagus and the lower oesophageal sphincter (LES) [Palmer, 2005]. The larynx extends from the epiglottis to the posterior portion of the cricoid cartilage. The trachea starts at inferior border of the cricoid cartilage. The posterior portion of the trachea does not contain cartilage which allows for oesophageal movement during swallowing [Chipas et al, 2005]. The cricoid cartilage is the only complete ring which should allow for compression of the oesophagus.

\section{REGURGITATION AND ASPIRATION}

There are three barriers that protect the patient against aspiration. Normal intragastric pressure (IGP) ranges between 5 and $10 \mathrm{mmHg}$. The LES is the first barrier to the regurgitation of gastric contents into the oesophagus. It normally maintains a pressure of $25 \mathrm{mmHg}$. If there is a decrease in LES pressure and/or increase in IGP, sufficient to cause a breach of the LES, then regurgitation of gastric contents into the oesophagus occurs [Brimacombe and Berry, 1997; Apfel and Roewer, 2005]. Most anaesthesia related medications result in a decrease in LES which may allow gastric contents to enter the body of the oesophagus [Cotton and Smith, 1984].

The UES is the second line of defense against aspiration. The UES generally maintains a pressure of approximately $40 \mathrm{mmHg}$. The induction of general anaesthesia results in an impressive drop to less than $10 \mathrm{mmHg}$ [Vanner et al, 1992]. Once UES is decreased to this level regurgitated material may have access to the pharynx.

The final line of defense is the patient's protective laryngeal reflexes. Normally patients are able to protect themselves against the aspiration of gastric contents. The induction of general anaesthesia results in depressed or absent protective pharyngeal reflexes [Odom-Forren and Watson, 2005]. General anaesthesia has profound impact on the LES, UES and protective pharyngeal reflexes. Aspiration is of particular concern for at-risk patients.

The overall incidence of aspiration, during general anaesthesia, is approximately 1:2,000-3,000. For paediatric patients the incidence is $1: 1,200-2,600$. The incidence of aspiration increases by three to four times in the context of emergency surgery. Aspiration is simply liquid or particulate matter entering the lungs through passive regurgitation or active vomiting in the setting of diminished/absent protective pharyngeal reflexes. There are three types of aspiration pneumonia. Aspiration pneumonia related to gastric acid aspiration results in a direct chemical insult to the lung tissue. The extent of damage is dependent upon the volume and $\mathrm{pH}$ of the contents aspirated. The first phase of damage is immediate and related to the destruction of lung epithelium. The second phase occurs $2-3 \mathrm{~h}$ later and is the result of an inflammatory reaction. Symptoms range from no sequella to respiratory failure. Aspiration pneumonia related to the aspiration of bacteria in oropharyngeal or gastric contents is the second type. Many of the signs and symptoms are typical of pneumonia. The third type of aspiration pneumonia is related to aspiration of particulates. This will result in acute hypoxaemia and atelectasis [Janda et al, 2006].

\section{CRICOID PRESSURE}

The induction and maintenance of general anaesthesia results in a diminished UES, diminished LES and diminished/absent protective pharyngeal reflexes, potentially predisposing the patient to aspiration (Fig. 1). Cricoid pressure is the occlusion of the upper oesophagus by the application of pressure to the cricoid cartilage. This act will apply backward pressure and occlude the oesophagus between the cricoid ring and cervical vertebrae [Salem et al, 1974]. Thus cricoid pressure compensates for the loss of the UES.

The measurement of pressure is expressed in Newtons' (N) since it involves force. It takes $9.81 \mathrm{~N}$ to equal $1 \mathrm{~kg}$. Research has found that the application of $30-40 \mathrm{~N}$ is a sufficient amount of force for effective cricoid pressure [Wraight et al, 1983; Vanner and Pryle, 1992; Vanner et al, 1992]. $20-40 \mathrm{~N}$ is equivalent to $2-4.1 \mathrm{~kg}$ or 4.4-9.02 lbs of pressure. For the sake of convenience $1-2 \mathrm{~kg}$ of cricoid pressure for the conscious patient and $3-4 \mathrm{~kg}$ in the unconscious patient should be sufficient for effective cricoid pressure [Koziol et al, 2000].

The application of cricoid pressure is a simple three step process (Table 1). The first step is to locate the cricoid cartilage. The cricoid cartilage is the first complete cartilaginous ring located immediately below the thyroid cartilage (Fig. 2). Place the thumb and second finger on either side of the cricoid cartilage. Step two is the application of direct, downward pressure (Fig. 3). Care must be taken to avoid lateral displacement. Prior to the induction of anaesthesia cricoid pressure should be maintained at $10-20 \mathrm{~N}$ or $1-2 \mathrm{~kg}$. The amount of force exerted should not exceed $20 \mathrm{~N}$ or $2 \mathrm{~kg}$ [Janda et al, 2006; Jevon, 2006; Koziol et al, 2000]. Conscious volunteers can tolerate only $20 \mathrm{~N}$ or $2 \mathrm{~kg}$ of pressure before retching occurs [Vanner, 1992]. Results of a small study found that the application of cricoid pressure in awake volunteers paradoxically decreases LES [Brimacombe and Berry, 1997]. After loss of consciousness the force should 


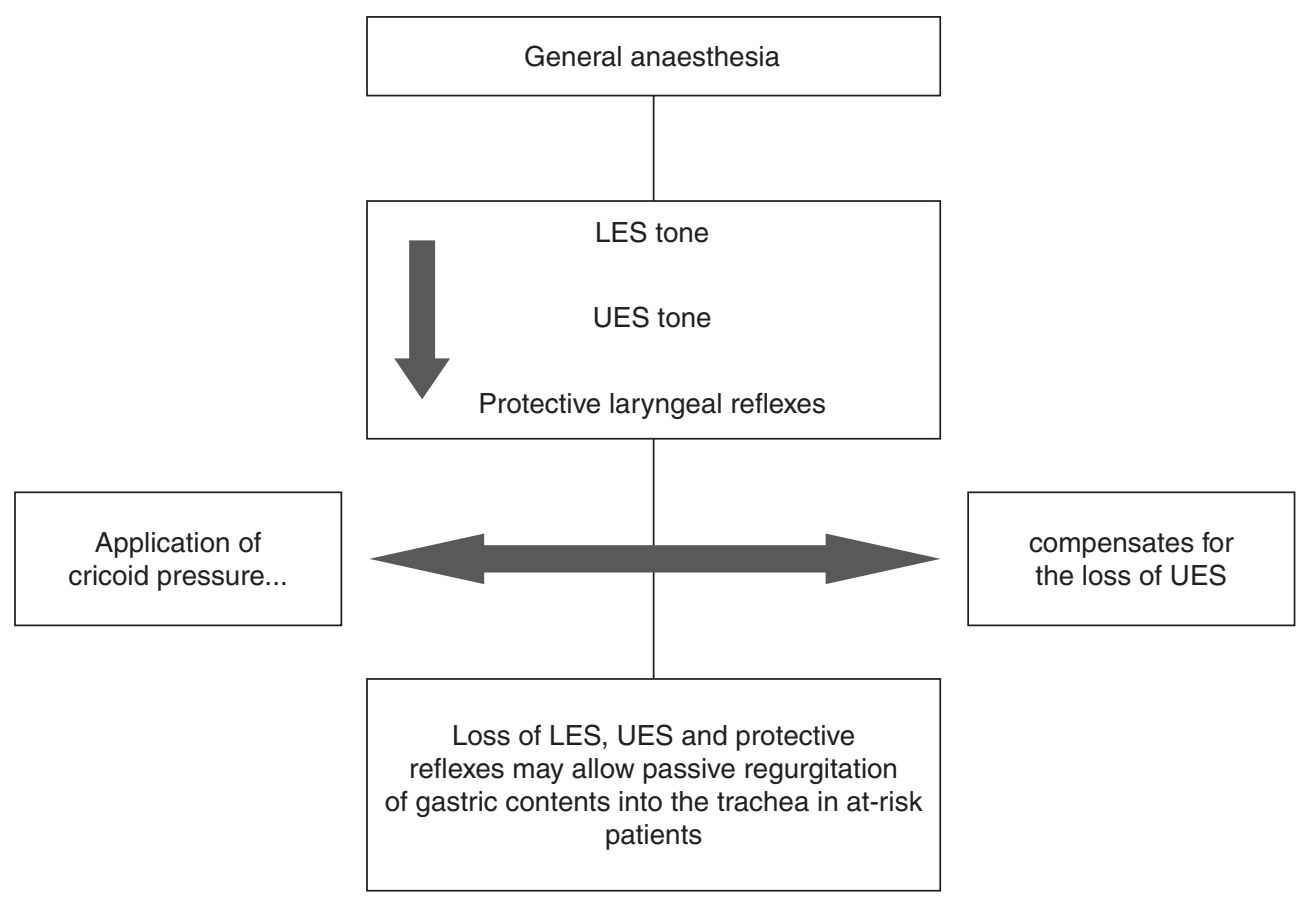

Figure 1. The use of cricoid pressure to compensate for the loss of the UES.

Table 1. The three step process for the application of cricoid pressure

1. Locate: The cricoid cartilage is immediately below the thyroid cartilage.

Place the thumb and second finger on either side of the cricoid cartilage.

2. Apply: Direct downward pressure. Do not deviate to the side.

$1-2 \mathrm{~kg}$ of pressure in the conscious patient. Never exceed $2 \mathrm{~kg}$.

Increase pressure once the patient loses

consciousness.

$3-4 \mathrm{~kg}$ of pressure in the unconscious patient. Never exceed $4 \mathrm{~kg}$.

3. Release: Only when instructed to do so.

Compiled from the following resources: Vanner [1992]; Jevon [2006]; Janda et al [2006]; Koziol et al [2000].

be increased to 30-40 N or 3-4 kg [Vanner, 1992; Janda et al, 2006; Jevon, 2006; Koziol et al, 2000]. The application of cricoid pressure should not exceed $40 \mathrm{~N}$ or $4 \mathrm{~kg}$. The third step is releasing pressure. Pressure should be maintained until the anaesthesia provider has secured the airway with an endotracheal tube and communicates to the assistant that he/she can release pressure [Janda et al, 2006; Jevon, 2006; Koziol et al, 2000]. For patients with suspected cervical spine injuries a two

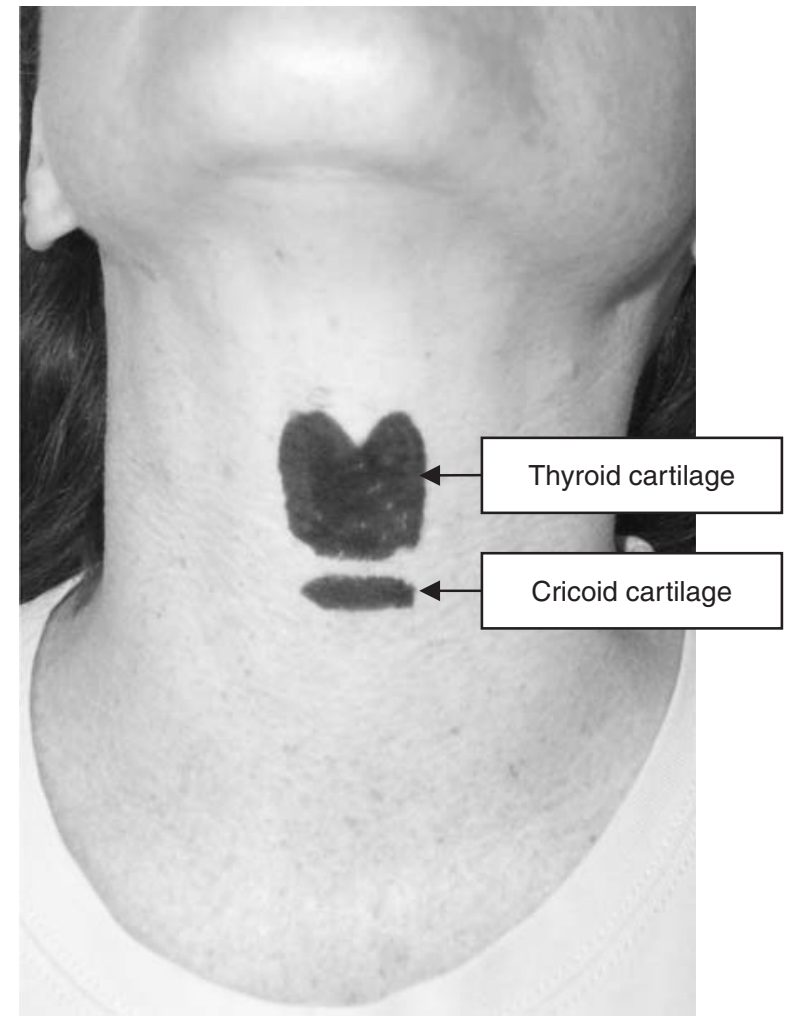

Figure 2. Location of cricoid cartilage. 


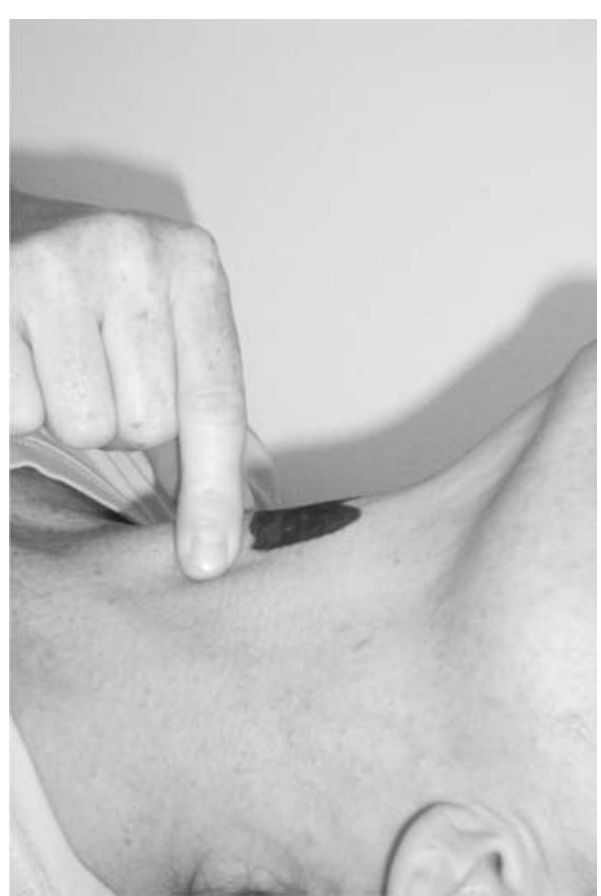

Figure 3. The application of cricoid pressure.

handed technique has been suggested [Hartely, 1993]. Extreme care to avoid head and neck movement must be taken during intubation of these patients.

\section{CONTROVERSY CONCERNING CRICOID PRESSURE}

Cricoid pressure has become an accepted technique to reduce the incidence of pulmonary aspiration in 'at-risk' patients. However several reports have began to question this practice. Brimacombe and Berry [1997] stated that the use of cricoid pressure has not been proven to reduce aspiration. In fact there is evidence that it can be ineffective and detrimental, resulting in difficult intubations and regurgitation. This sentiment was echoed by a review of the literature by Butler and Sen [2005] and Apfel and Roewer [2005].

Part of the controversy may stem from the fact that there is a relatively low incidence of pulmonary aspiration in patients undergoing general anaesthesia. It is also a well known fact that cricoid pressure is often applied ineffectively which may account for instances of difficult intubation, regurgitation and subsequent aspiration. Finally, it would be unethical to conduct a large prospective clinical trial and assign patients, at risk for pulmonary aspiration, to a group that would not have cricoid pressure applied.

\section{INDICATIONS FOR CRICOID PRESSURE APPLICATION}

The primary indication for cricoid pressure is to prevent aspiration of gastric contents. Patients at risk for aspiration may have diminished/altered laryngeal protective reflexes, anatomical or mechanical alterations and other causes. The inability to protect the airway due to diminished/absent laryngeal protective reflexes may be due to brain injuries, intoxication and neurological impairment. Anatomical or mechanical alterations include gastrointestinal obstructions, gastroesophageal reflux and conditions that affect gastric emptying. Other causes include pregnancy 2 nd-3rd trimester, the elderly, the difficult airway and emergency surgeries [Apfel and Roewer, 2005; Janda et al, 2006]. A secondary indication for the application of cricoid pressure is to reduce gastric insufflation of air during positive pressure ventilation with a mask. This application receives less attention. Cricoid pressure has been found to be effective for both adult and paediatric patients [Petito and Russell, 1988; Moynihan et al, 1993].

Some have mistakenly suggested that the application of cricoid pressure may help bring the glottis into view during a difficult intubation [Koziol et al, 2000; Jevon, 2006]. Cricoid pressure can actually make intubation more difficult. Applying pressure more cephalad, to the thyroid cartilage, will help in visualisation of the glottis [Roth, 2007].

\section{COMPLICATIONS AND CONTRAINDICATIONS}

Complications associated with cricoid pressure are rare. There has been a report of a fractured cricoid cartilage after the application of cricoid pressure in a patient with a history of a previous laryngeal trauma [Heath et al, 1996]. Complete airway obstructions have been reported in patients with undiagnosed laryngeal trauma and undiagnosed supraglottic mass [Shorten et al, 1991; Georgescu et al, 1992]. Excessive cricoid pressure may result in a complete airway obstruction in normal patients [Allman, 1995]. Rupture of the oesophagus can occur with the application of cricoid pressure during active vomiting [Ralph and Wareham, 1991]. The act of vomiting can generate IGPs greater than $100 \mathrm{mmHg}$ 
[Apfel and Roewer, 2005] which can result in oesophageal tears. Contraindications to the application of cricoid pressure include active vomiting, traumatic injuries to the anterior neck, suspected laryngeal trauma, suspected supraglottic masses and unstable cervical spine injuries [Hein and Owen, 2005].

\section{PROBLEMS WITH COGNITIVE AND PRACTICAL APPLICATION OF CRICOID PRESSURE}

Despite being an accepted and common technique several studies have shown that many clinicians can not identify and apply the correct of amount of pressure. In addition some clinicians are unable to identify the correct anatomical landmarks to apply cricoid pressure.

A study designed to assess the cognitive knowledge of the recommended amount of pressure and the practical application of cricoid pressure was undertaken. A convenience sample of 102 perioperative nurses were included as participants. Data was collected in the form of a questionnaire that assessed cognitive knowledge. Practical application was assessed with the use of a lifesize laryngotracheal model mounted on an infant scale. The correct amount of cricoid pressure was reported by $5 \%$ of the participants and $95 \%$ either overestimated or underestimated the correct amount of pressure. Practical application of the appropriate amount of cricoid pressure was demonstrated by $13 \%$ of the participants. Most participants applied too much pressure $(69.5 \%)$. It was noted by the researchers that a large number of the participants were unable to correctly identify the cricoid cartilage on the anatomical model [Koziol et al, 2000].

A small study of 29 anaesthetic practitioners and anaesthetists were assessed on the application of cricoid pressure. It was found that approximately $31 \%$ of the practitioners applied the correct amount of cricoid pressure. The majority, $55 \%$, applied too much pressure while $13 \%$ applied insufficient pressure [Donnellon, 2003].

A mailed questionnaire to anaesthetists in the UK found that cricoid pressure was routinely used but varied in how it is applied [Morris and Cook, 2001]. Anaesthesia assistants in the UK were interviewed about cricoid pressure. It was found that approximately $33 \%$ could quote the correct amount of pressure that should be applied. Less than $50 \%$ could list one contraindication to the application of cricoid pressure. Most of the anaesthetic assistants had not been formally trained with a model prior to using it clinically. In addition the technique varied among participants [Meek et al, 1999]. In a French questionnaire only $52 \%$ of the anaesthesiologists that regularly use cricoid pressure could give an adequate description of the technique [Benhamou, 1993]. In a Swedish study $40 \%$ of the anaesthesiologists were unaware of any contraindications to cricoid pressure [Schmidt and Akeson, 2001].

The application of cricoid pressure is also routinely performed in the emergency department (ED). A recent study assessed the application of cricoid pressure by ED staff. The study included 38 physicians and 69 nurses. A life-size laryngotracheal model was placed on a scale. Each participant was blinded to the amount of pressure exerted and were allowed five attempts. The mean value of the five attempts was calculated. It was found that 27 participants or $25 \%$ were able to apply the correct amount of cricoid pressure. Of the 80 participants who applied the incorrect amount of pressure $63 \%$ were below the target force of $3.060 \mathrm{~kg}$ and $37 \%$ were above the target force of $4.075 \mathrm{~kg}$ [Clark and Trethewy, 2005]. A statistically significant difference was noted between nurses in the ED and the perioperative nurses from the Koziol et al [2000] study. However, this assertion is not accurate. There was a significant difference in how each study was conducted. The participants in the Clark and Trethewy [2005] study had five attempts at reaching the target range, though all values were combined into a mean. In the Koziol et al [2000] study each perioperative nurse had only once chance to apply cricoid pressure.

Several studies have shown that clinicians, whether physician or nurse, have cognitive deficits concerning the application of cricoid pressure. There appears to be a wide variation in the force that is applied and techniques of application.

\section{TEACHING TECHNIQUES}

It is apparent that most clinicians would benefit from additional training. A quality improvement (QI) programme to educate operating room nurses on the correct application of cricoid pressure was reported recently. Fifty-one operating room nurses who routinely apply cricoid pressure were included. Training concentrated on didactic as well as hands on training. The nurses were given a pre-test and were asked to apply cricoid pressure on a 'life-sized' laryngotracheal model and the amount of pressure was recorded. This was followed by an educational programme, that covered basic anatomy, physiology of regurgitation, application of the correct amount of pressure and when it should be initiated and released. All the participants were allowed to practice on the model and visualise the amount of pressure 
that should be applied. The post-test was the same as the pre-test. It was found that participants vastly improved on the didactic questions as well as hands on testing. Only $2 \%$ of the participants answered all the pretest questions correctly compared to $76.5 \%$ after training. Hands on application during the pre-test found that only $3.9 \%$ of the participants could correctly identify the correct anatomical location for the application of cricoid pressure and apply the correct pressure. After the educational programme, $68.6 \%$ of the participants were successful in locating the correct anatomical location and applying the correct pressure [Patten, 2006].

A similar technique also utilised a laryngotracheal model and an infant scale to measure the amount of pressure applied. The 'trainees' were first to apply cricoid pressure while being blinded to the amount of pressure being applied. Next the participants were instructed on the correct amount of pressure and were asked to apply pressure again. The participants were allowed to practice applying the correct amount of pressure until they felt that they could reliably replicate the recommended amounts of pressure. The participants demonstrated retention of the cricoid pressure skills 3 months later [Herman et al, 1996].

A more sophisticated approach utilised a part task trainer to educate clinicians in the correct application of cricoid pressure. A 'life-size' mannequin was equipped with pressure sensors to detect the amount of pressure being applied and microswitches to identify the direction of pressure. Data was collected from 50 clinicians of whom $92 \%$ stated that they apply cricoid pressure on a regular basis and $52 \%$ taught its application. Prior to training none of the participants could identify the correct amount of pressure that should be applied, $24 \%$ were unable to identify the correct anatomical landmark and only $10 \%$ applied the correct amount of pressure. After training $100 \%$ identified the correct amount of pressure that should be applied, $100 \%$ could correctly identify the cricoid cartilage and $90 \%$ applied the correct amount of pressure [Owen et al, 2002].

A relatively low tech approach to achieve clinically effective cricoid pressure can be accomplished by the use of a $50 \mathrm{~mL}$ air filled syringe. Kopka and Robinson [2005] found that by occluding the outlet of the syringe and depressing it from the $50 \mathrm{~mL}$ mark down to $33 \mathrm{~mL}$ equals $3.06 \mathrm{~kg}$ or $30 \mathrm{~N}$. A convenience sample of 64 anaesthetic assistants were recruited and divided into two groups. It was found that only $17 \%$ of the participants had cognitive knowledge concerning the correct amount of pressure. The first group was asked to apply pressure to the syringe to simulate the pressure that would be applied for cricoid pressure. Only 19\% applied the correct amount of pressure. The second group was trained on the syringe and it was found that $47 \%$ were able to reproduce the correct amount of pressure. The authors concluded that a brief 'training' session before the application of cricoid pressure may result in the application of effective cricoid pressure. Drawbacks of this training technique are that it may lack the 'clinical feel' of a patient and it does not appear as effective as other training techniques.

Additional techniques devised to teach the correct amount of cricoid pressure have included self-applied pressure to the nose or the cricoid. Concerning the nose it has been suggested that applying enough pressure to cause pain would approximate the correct amount of cricoid pressure. This however is incorrect. It also has been suggested that applying enough pressure to prevent swallowing should approximate the correct amount of pressure of cricoid pressure [Hein and Owen, 2005]. However it only takes $20 \mathrm{~N}$ of pressure to cause retching in conscious patients [Vanner, 1992]. So if one practices pressure on ones own cricoid he/she would never reach the correct amount of pressure without retching. Neither of these techniques are useful in teaching the correct amount of pressure to apply.

These studies imply that formalised training sessions are beneficial in teaching important background cognitive knowledge that can be applied to the practical skill of applying cricoid pressure. Only after formalised training can the clinician apply cricoid pressure in a knowledgeable, correct and effective manner (Table 2). Concern about ineffective cricoid pressure has been recently raised by two informative articles in the UK. Both have suggested the need for the development of clinical guidelines, formalised training and routine competency testing [Gardiner and Grindrod, 2005; Stanton, 2006]. Before the dismissal of cricoid pressure as an ineffective manoeuvre there should be formalised training in its correct application. Only after it is applied correctly can a critical evaluation determine if it is a clinically relevant technique.

\section{CONCLUSION}

Clinicians responsible for the application of cricoid pressure should have a thorough knowledge of basic anatomy, physiology of regurgitation, indications for application and common problems/mistakes when applying cricoid pressure. The practical application of the correct amount of cricoid pressure is especially important to help the clinician apply an effective amount of force to occlude the oesophagus. For teaching and training purposes most healthcare facilities have access to an infant scale and a human model of the laryngotracheal 
Table 2. Suggested teaching components for cricoid pressure training

- Didactic component

(a) Basic anatomy as it relates to cricoid pressure

(b) Basic physiology of regurgitation and aspiration

(c) Description of the application of cricoid pressure

(d) Indications for cricoid pressure

(e) Complications and contraindications

(f) Why training is necessary

- Hands on practice

(a) Identification of anatomy

(b) Application of the correct pressure for the conscious and unconscious patient

- Post-test includes recall of basic facts and practical application

- Competency testing at regular timed intervals

Compiled from the following resources: Koziol et al [2000]; Clark and Trethewy [2005]; Patten [2006]; Herman et al [1996]; Gardiner and Grindrod [2005]; Stanton [2006]. anatomy. This can be a simple and effective training tool. It is important to have routine competency testing, at timed intervals, to assess how effectively clinicians are applying cricoid pressure.

\section{ACKNOWLEDGEMENTS}

Sue Echols, Anne Heimann and Cathy Collett from the Bryan/LGH College of Health Sciences library were instrumental in procuring required articles for this manuscript. Editorial assistance was provided by Sandra Rosse, Clinical Research Analyst at Good Samaritan Hospital, Kearney, Nebraska. The author also wishes to thank his wife Dianne, daughter Katelyn, Tessa, Ethan and Treu for allowing for some quiet time to work on this manuscript.

\section{REFERENCES}

Allman KG. The effect of cricoid pressure application on airway patency. Journal of Clinical Anesthesia 1995; 7(3): 197-199.

Apfel CC, Roewer N. Ways to prevent and treat pulmonary aspiration of gastric contents. Current Opinion in Anaesthesiology 2005; 18(2): 157-162.

Benhamou D. French obstetric anesthetists and acid aspiration prophylaxis. European Journal of Anaesthesiology 1993; 10(1): 27-32.

Brimacombe JR, Berry A. Cricoid pressure. Canadian Journal of Anaesthesia 1997; 44(4): 414-425.

Butler J, Sen A. Cricoid pressure in emergency rapid sequence induction. Emergency Medicine Journal 2005; 22(11): 815-816.

Chipas A, Ellis, Zaglaniczny KL. Airway management, Chapter 20. In: Nagelhout JJ, Zaglaniczny KL (eds). Nurse Anesthesia (3rd edn). USA: Elsevier Saunders, 2005.

Clark RK, Trethewy CE. Assessment of cricoid pressure application by emergency department staff. Emergency Medicine Australasia 2005; 17(4): 376-381.
Cotton BR, Smith G. The lower oesophageal sphincter and anaesthesia. British Journal of Anaesthesia 1984; 56(37): 37-46.

Donnellon M. Survey of knowledge and application of cricoid pressure. Technic. The Journal of Operating Department Practice 2003; 236: 14-16.

Gardiner E, Grindrod E. Applying cricoid pressure. British Journal of Perioperative Nursing 2005; 15(4): 164-168.

Georgescu A, Miller JN, Lecklitner ML. The Sellick Maneuver causing complete airway obstruction. Anesthesia and Analgesia 1992; 74(3): 457-459.

Hartely M. Cricoid pressure and potential cervical spine injuries. Anaesthesia 1993; 48(12): 1113.

Heath KJ, Palmer M, Fletcher SJ. Fracture of the cricoid cartilage after Sellick's manoeuvre. British Journal of Anaesthesia 1996; 76(6): 877-878.

Hein C, Owen H. The effective application of cricoid pressure. Journal of Emergency Primary Health Care 2005; 3(1-2) (jephc.com).
Herman NL, Carter B, Van Decar TK. Cricoid pressure: teaching the recommended level. Anesthesia and Analgesia 1996; 83(4): 859-863.

Janda M, Scheeren TWL, NoldgeSchomburg GFE. Management of pulmonary aspiration. Best Practice and Research Clinical Anaesthesiology 2006; 20(3): 409-427.

Jevon P. Cricoid pressure. Nursing Times 2006; 102(39): 26-29.

Kopka A, Robinson D. The $50 \mathrm{ml}$ syringe training aid should be utilized immediately before cricoid pressure application. European Journal of Emergency Medicine 2005; 12(4): 155-158.

Koziol CA, Cuddeford JD, Moos DD. Assessing the force generated with application of cricoid pressure. AORN Journal 2000; 72(6): 1018-1030.

Meek T, Gittins N, Duggan JE. Cricoid pressure: knowledge and performance amongst anaesthetic assistants. Anaesthesia 1999; 54(1): 59-62.

Morris J, Cook TM. Rapid sequence induction: a national survey of practice. Anaesthesia 2001; 57(4): 1090-1097. 
Moynihan RJ, Brock-Utne JG, Archer JH, Feld LH, Kreitzman TR. The effect of cricoid pressure on preventing gastric insufflation in infants and children. Anesthesiology 1993; 78(4): 652-656.

Odom-Forren J, Watson D. History of moderate sedation. In: Odom-Forren J, Watson D (eds). Practical Guide to Moderate Sedation/Analgesia (2nd edn). St. Louis, MO: Mosby/Elsevier, 2005.

Owen H, Follows V, Reynolds KJ, Burgess G, Plummer J. Learning to apply effective cricoid pressure using a part task trainer. Anaesthesia 2002; 57(11): 1098-1101.

Palmer TJ. Hepatobiliary and gastrointestinal disturbances, Chapter 28. In: Nagelhout JJ, Zaglaniczny KL (eds). Nurse Anesthesia (3rd edn). USA: Elsevier Saunders, 2005.

Patten SP. Educating nurses about correct application of cricoid pressure. AORN Journal 2006; 84(3): 449-461.
Petito SP, Russell WJ. The prevention of gastric inflation - a neglected benefit of cricoid pressure. Anaesthesia and Intensive Care 1988; 16(2): 139-143.

Ralph SJ, Wareham CA. Rupture of the oesophagus during cricoid pressure. Anaesthesia 1991; 46(1): 40-41.

Roth JV. Cricoid pressure is for full stomachs, thyroid pressure is for assisting intubations. Anesthesia and Analgesia 2007; 104(1): 219.

Salem MR, Sellick BA, Elam JO. The historical background of cricoid pressure in anesthesia and resuscitation. Anesthesia and Analgesia 1974; 53(2): 230-232.

Schmidt A, Akeson J. Practice and knowledge of cricoid pressure in southern Sweden. Acta Anaesthesiologica Scandinavia 2001; 45(10): 1210-1214.

Shorten GD, Allfille PH, Gliklich RE. Airway obstruction following the application of cricoid pressure. Journal of Clinical Anesthesia 1991; 3(5): 403-405.
Stanton J. Literature review of safe use of cricoid pressure. Journal of Perioperative Practice 2006; 16(5): 250-257.

Vanner RG. Tolerance of cricoid pressure by conscious volunteers. International Journal of Obstetric Anesthesia 1992; 1(4): 195-198.

Vanner RG, Pryle BJ. Regurgitation and oesophageal rupture with cricoid pressure: a cadaver study. Anaesthesia 1992; 47(9): 732-735.

Vanner RG, O’Dwyer JP, Reynolds F. Upper oesophageal sphincter pressure and the effect of cricoid pressure. Anaesthesia 1992; 47(2): 95-100.

Wraight WJ, Chamney AR, Howells TH. The determination of an effective cricoid pressure. Anaesthesia 1983; 38(5): 461-466. 